\title{
Design and development of a long-travel positioning actuator and tandem constant force actuator safety system for the Hobby Eberly Telescope Wide Field Upgrade
}

\author{
Nicholas T. Mollison ${ }^{\mathrm{a} \dagger}$, Jason R. Mock ${ }^{\mathrm{a}}$, Ian M. Soukup ${ }^{\mathrm{a}}$, Timothy A. Beets ${ }^{\mathrm{a}}$, John M. Good ${ }^{\mathrm{b}}$, Joseph \\ H. Beno ${ }^{a}$, Herman J. Kriel ${ }^{b}$, Sarah E. Hinze ${ }^{a}$, Douglas R. Wardell ${ }^{a}$, James T. Heisler ${ }^{a}$ \\ ${ }^{a}$ The University of Texas at Austin, Center for Electromechanics, 1 University Station R7000, \\ Austin, Texas USA 78712 \\ ${ }^{\mathrm{b}}$ The University of Texas McDonald Observatory, 1 University Station C1402, Austin, Texas, USA \\ 78712-0259;
}

\begin{abstract}
The Wide Field Upgrade presents a five-fold increase in mass for the Hobby-Eberly Telescope's ${ }^{*}$ tracker system. The design of the Hobby-Eberly Telescope places the Prime Focus Instrument Package (PFIP) at a thirty-five degree angle from horizontal. The PFIP and its associated hardware have historically been positioned along this uphill axis (referred to as the telescope's Y-axis) by a single screw-type actuator. Several factors, including increased payload mass and design for minimal light obscuration, have led to the design of a new and novel configuration for the $\mathrm{Y}$-axis screw-drive as part of the tracker system upgrade. Typical screw-drive designs in this load and travel class (approximately 50 kilonewtons traveling a distance of 4 meters) utilize a stationary screw with the payload translating with the moving nut component. The new configuration employs a stationary nut and translating roller screw affixed to the moving payload, resulting in a unique drive system design. Additionally, a second cable-actuated servo drive (adapted from a system currently in use on the Southern African Large Telescope) will operate in tandem with the screw-drive in order to significantly improve telescope safety through the presence of redundant load-bearing systems. Details of the mechanical design, analysis, and topology of each servo drive system are presented in this paper, along with discussion of the issues such a configuration presents in the areas of controls, operational and failure modes, and positioning accuracy. Findings and results from investigations of alternative telescope safety systems, including deformable crash barriers, are also included.
\end{abstract}

Keywords: Center for Electromechanics, Hobby-Eberly Telescope, HETDEX, University of Texas, telescope safety, precision positioning systems, actuators

\section{INTRODUCTION}

The Hobby Eberly Telescope (HET) (Figure 1) is undergoing a major upgrade in preparation for the HET Dark Energy eXperiment ${ }^{\ddagger}$ (HETDEX), as well as to enhance support capabilities for current and future instrumentation ${ }^{1}$. HETDEX entails two major hardware upgrades to the HET: the Wide Field Upgrade (WFU), and the fabrication and installation of 192 spectrometers called VIRUS ${ }^{2}$. The WFU consists of four related engineering projects: 1) Replacing the current spherical aberration corrector with the Wide Field Corrector ${ }^{3}$ (WFC), which will increase the HET field of view from 4' to $\left.22^{\prime}, 2\right)$ replacing the entire tracker system, 3) a new Prime Focus Instrument Package (PFIP), and 4) modifications to the HET facility ${ }^{1}$. Under the tracker portion of the program, improvements are being made to the HET's motion control systems to increase tracker payload capacity and to hasten "reset" speed, allowing more observations per night as required for HETDEX ${ }^{2}$.

\footnotetext{
* The Hobby-Eberly Telescope is operated by McDonald Observatory on behalf of the University of Texas at Austin, the Pennsylvania State University, Stanford University, Ludwig-Maximillians-Universität München,and Georg-August-Universität, Göttingen

† R.S.: email: n.mollison@cem.utexas.edu

* http://hetdex.org/
} 

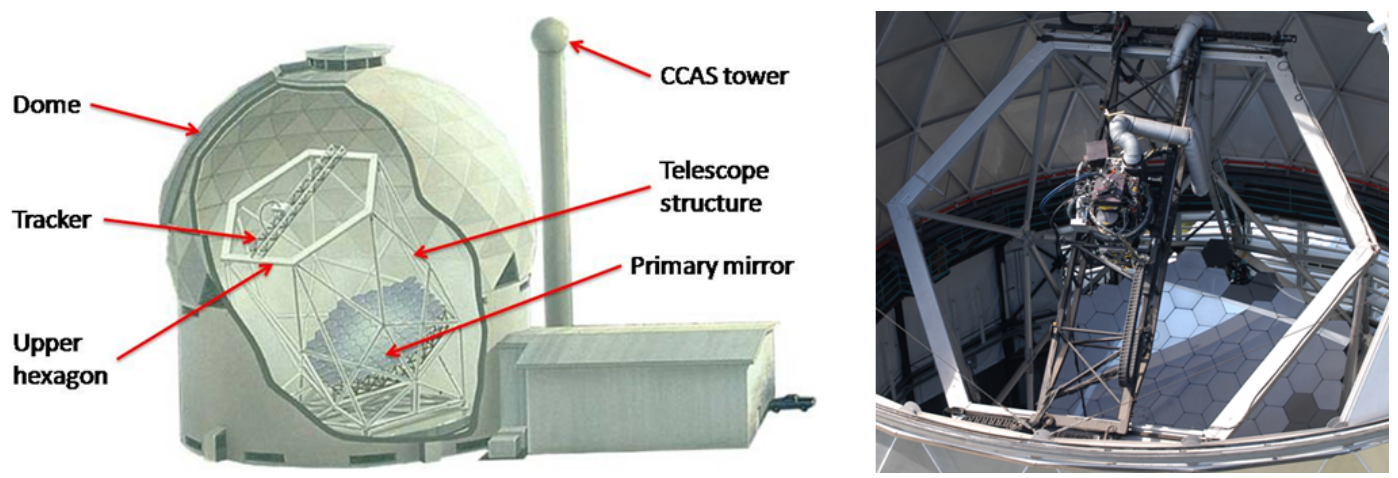

Figure 1. Left: The Hobby Eberly Telescope. Right: The existing tracker, comprising the bridge and all components mounted to the telescope upper hexagon. The Prime Focus Instrument Package and associated components that constitute the tracker payload are near the center of the bridge structure, which they translate along over a total distance of four meters.

The HET tracker system utilizes a total of nine linear actuators to position the PFIP such that it follows an astronomical object's trajectory in real-time, while simultaneously maintaining precise alignment with the spherical focal surface of the $11 \mathrm{~m}$ primary mirror array. These axes of motion are described in Figure 2.
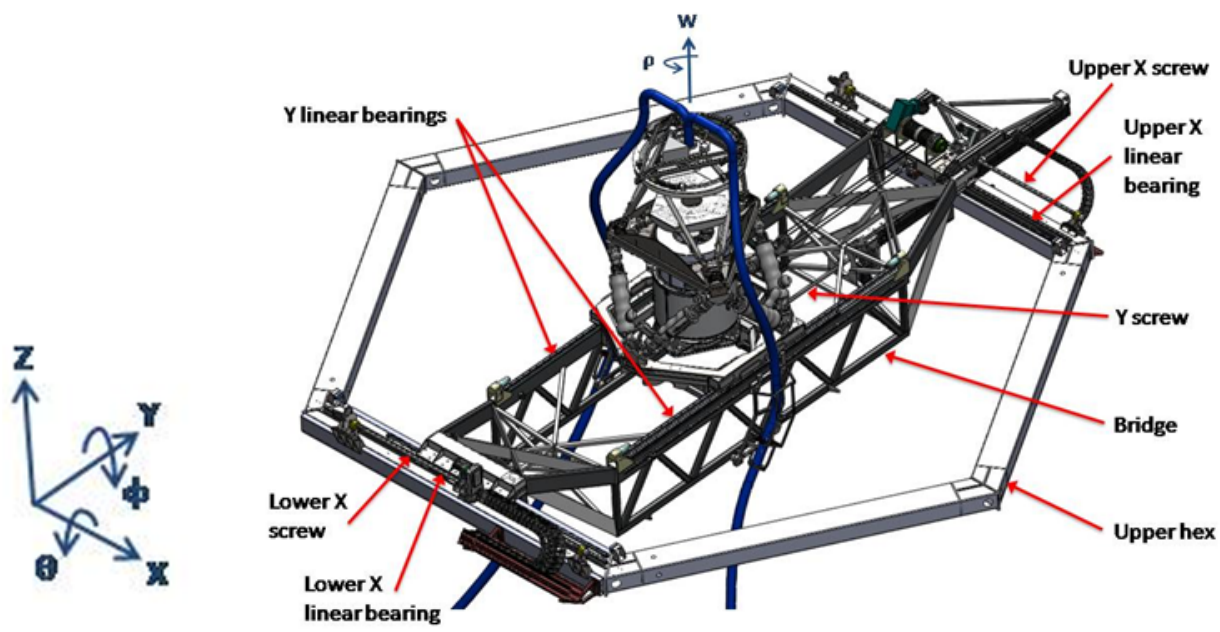

Figure 2. The new tracker design for the Wide Field Upgrade is illustrated. Like the existing tracker, large displacement linear motions along the $\mathrm{X}$ and $\mathrm{Y}$ axes are performed by linear roller screw drives. Two actuators, one on each side of the HET upper hexagon, position the bridge structure in the X direction, and a single actuator positions the PFIP and its hexapod and supporting platform in Y. Another six linear actuators are configured in a Stewart platform (hexapod) arrangement to control the smaller displacement tip (theta), tilt (phi), and $\mathrm{Z}$ axis motions required to follow a trajectory over a spherical surface.

The Wide Field Upgrade presents a five-fold increase in mass for the Hobby-Eberly Telescope's tracker system, with the total mass of the new tracker approaching 19,000kg. The design of the Hobby-Eberly Telescope places the PFIP at a thirty-five degree angle from horizontal ${ }^{1}$. The PFIP, WFC, hexapod, and associated sub-structure and hardware (collectively referred to as the "payload" within this paper) have historically been positioned along this uphill Y-axis by a single screw-type actuator. Several factors, including the substantial increase in payload mass and design for minimal light obscuration, have led to a Y-axis screw-drive design which is significantly different from that of the existing tracker.

Introducing a new drive system design subsequently prompted re-examination of telescope hardware failure modes and effects, and corresponding preventive safety measures. Several concepts for new safety systems were proposed with a total of three selected for further development, each capable of providing an additional layer of safety over the current tracker system. Energy absorbing crash barriers (bump stops) and hydraulically actuated linear brakes embody "standby" emergency systems; and an independent force-controlled servo drive system acting in parallel with the Y-axis screw- 
drive constitutes a redundant system. The mechanical design, analysis, and topology of the tandem drive system deployed on the new tracker will be presented along with commentary on the critical design decisions in order to provide a complete view of their integrated engineering and development.

\section{Y-AXIS POSITIONING DRIVE DESIGN}

\subsection{Overview of the existing HET drive system}

Discussion of the existing HET Y-axis screw-drive system is necessary in order to preface the technical challenges and design decisions generated by the Wide Field Upgrade. With the exception of a few hardware and software issues which were exposed and consequently resolved during commissioning on the HET, the existing Y-axis drive has a strong record of reliable operation (likewise can be said for the similar Southern African Large Telescope [SALT] design). This proven history warranted that its topology should serve as a familiar and feasible starting point for the upgraded tracker design.

The mechanical design of the HET tracker and its motion systems are based on kinematic design principles, detailed discussion of which can be found in references 4 and 5. An introductory description of the Y-axis motion system follows: The PFIP and hexapod are mounted to the rigid structure of the Lower Hexapod Frame (LHF). The LHF travels along the bridge in the Y-axis direction on linear ball bearing guides mounted atop each longitudinal box beam section or "rail" of the bridge truss. One pair of linear bearing blocks shares a common bearing rail and supports the LHF on one side of the bridge, while a third linear bearing with a cross-slide bearing component supports the LHF at a single mounting point on the opposite side of the bridge. The cross-slide provides a degree of freedom in the X-axis such that misalignment errors between the parallel $\mathrm{Y}$-axis linear bearings do not produce fluctuating mechanical stresses in the LHF. The Y-axis roller screw is affixed to the bridge on the same side as the pair of linear bearings, and is positioned directly above the bridge bearing rail.

Due to the orientation of the tracker bridge at a thirty five degree incline from horizontal, the Y-axis screw is loaded in a constant state of tension as it supports the downhill vector of the payload weight. The roller screw is mounted to the bridge with a "fixed-free" support bearing arrangement, whereby the upper end of the screw is held axially fixed by a duplex pair of angular contact bearings and the lower end is supported by radial bearings with an axial degree of freedom; therefore accommodating thermal expansion of the screw. A dual speed drive system translates the LHF up and down the length of the roller screw. The nut component of the screw-drive is coupled to the LHF through a direct drive motor rigidly mounted to the LHF structure. At the upper end of the screw, a motor and reduction gearbox are affixed to the bridge and coupled with the end of the screw shaft. Each motor is paired with a spring-applied, pneumaticallyreleased disc brake. Two modes of operation, slewing and tracking, are possible. In slewing mode, the roller screw is held stationary with one disc brake while the direct drive motor rotates the nut component. In tracking mode, the nut is held fixed with the other disc brake while the motor-gearbox rotates the roller screw (see reference 5 for detailed discussion).

This topology possesses many positive qualities that were desirable to retain in the design of a new tracker for the WFU, as well as several tradeoffs that have potential to negatively impact telescope performance. These tradeoffs consequently expose the limitations of the existing HET tracker design for use with significantly more massive payloads, and will be the focus of the next section.

\subsection{Y-axis drive system design considerations}

The collection of functional requirements and performance optimization goals for the WFU and HETDEX creates several design considerations which have proven to be deterministic factors in the drive system design. The most significant of these, in terms of influencing the overall design approach, is the natural frequency performance of the loaded bridge structure.

To begin, the existing HET design places many components, including the hexapod struts and wide field corrector, inside the volume of the bridge structure ${ }^{1}$. This impedes access for routine inspection and maintenance of these critical components as a side-effect; plus adapting this design for the new dimensions required by the WFU would produce a much wider structure, placing more of the bridge outside the WFC central obscuration area (the area where obstructions to incoming light have the least adverse effect). An approach similar to the SALT design is chosen for the tracker upgrade, which requires lowering the main bridge trusses such that the hexapod and PFIP remain above and outside the bridge volume. This design initially preserves the baseline approach of a single screw-drive mounted to one side of the 
bridge. The semi-kinematic linear bearing arrangement remains unchanged as well, with the exception of an additional bearing and cross-slide for a total of four linear bearings guiding the LHF in the Y-axis (Figure 3). The bearings have also been scaled up from THK model HSR35C and SR35T to higher load capacity THK SHS65LC.

Structural analysis of the bridge is heavily focused on rigidity and natural frequency performance. Although preliminary FEA simulations indicated WFU performance requirements could potentially be satisfied, the asymmetry of the semikinematic design and single screw-drive offset to one side of the bridge creates a significant challenge with the enormous moment loads that would be transmitted through one bridge truss member. Further detailed analysis of structural performance was conducted using a combination of SolidWorks Simulation (formerly Cosmos) and Algor software, and results were co-validated between the two. Resultant loading conditions produced unacceptable natural frequency behavior in the baseline bridge design (Figure 3). A correspondingly asymmetric, reinforced bridge design generated improvement, yet the drive-side bridge members would have to be widened to the point of creating excessive weight and obscuration in order to achieve the desired first-mode natural frequency of $>10 \mathrm{~Hz}$ for the loaded bridge (approximately a factor of $2 \mathrm{x}$ above the $5 \mathrm{~Hz}$ telescope structure first-mode to avoid disturbance coupling and resonance).

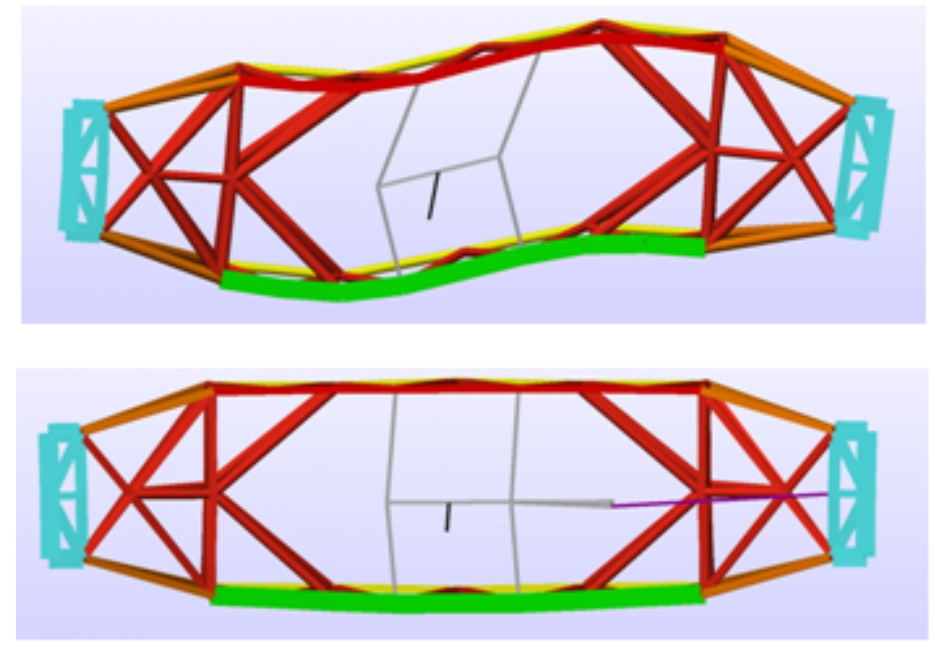

Figure 3. $8 \mathrm{~Hz}$ natural frequency and first mode shape of the bridge structure with an offset screw-drive (top) versus $12 \mathrm{~Hz}$ frequency and first mode shape of with the screw-drive located in the center of the bridge and coincident with the vertical plane through the payload center of mass (bottom).

It has been shown that orienting the Y-axis drive screw along one side of the bridge creates less than ideal performance tradeoffs for the WFU application. There is precious little left to optimize within the geometry of the structure without coincidentally infringing upon other functional requirements of the tracker; and iterative changes to material shape and size produced diminishing returns in performance versus penalties in weight and obscuration. Several alternative drive concepts aimed at mitigating the load conditions imposed on the bridge through the drive system were subsequently evaluated. Among these were rack and pinion gear-drive systems, tandem screws, cable actuated, and hydraulic systems. Ultimately, it was determined that the reliability, dynamic running accuracy, and successful operational history of a single screw-drive would remain the preferred solution if it could be deployed such that the longitudinal axis of the screw would lie on or very near the vertical plane through the payload center of mass. Several telescope integration challenges would have to be met as well. The next section will describe how these goals were achieved within the chosen design solution.

\subsection{Y-axis drive mechanical design}

Positioning the Y-axis roller screw coincident with the vertical plane of the payload center of mass excludes the option of a stationary (non-translating) screw mounted within the volume of the bridge, as it would be forced to overlap with either the physical operating space of the PFIP or the incoming light path to the WFC. Mounting a stationary screw outside of the bridge volume and away from these space constraints, i.e. fore or aft of the bridge in the Y-direction, creates impractical design requirements for screw support structures, tracker interface hardware, and integration within HET facilities. However, the inverse design solution of a roller screw which translates with its payload is able to occupy and reserve much of its volume to within the envelope of the telescope structure. Several iterations of this concept were 
produced and a comparative analysis concluded the system layout and location of components shown in Figure 4 best satisfies a multitude of tracker system requirements and design goals.

The fundamental dual-servo slew and track drive system detailed for the X-axis screws in reference 5 is replicated on the Y-axis, but with the following key differences:

- The tracking drive and "fixed bearing" end of the roller screw are coupled to the Lower Hexapod Frame and translate with the payload in the $\mathrm{Y}$-axis.

- The slew drive and roller screw nut remain stationary relative to the bridge.

- The segment of roller screw between the slew drive and tracking drive is loaded in a constant state of tension from the weight of the payload. Axial force on the Y-axis roller screw should never alternate direction, and should remain near constant during normal operation. Therefore, a one-direction spherical roller thrust bearing (SKF 29412-E) has been selected to couple the payload to the screw due to its advantageous running accuracy, screw deflection tolerance and self-aligning characteristics, bearing element rigidity, and axial load capacity.

- The "free bearing" end of the screw, opposite the tracking drive end, translates along a linear ball bearing guide rail and experiences no load other than the weight of the screw behind the slew drive, and reaction forces to bearing alignment error.

The Y-axis uses an SKF brand roller screw similar to the X-axis screws, with ground $60 \mathrm{~mm}$ diameter by $10 \mathrm{~mm}$ pitch threads and 6 satellite roller nut. However, the unidirectional Y-axis load permits the use of a non-preloaded solid planetary nut resulting in $\sim 10 \%$ greater efficiency (and therefore reduced net heat generation), and 1.6x greater load capacity versus an equivalent split preloaded nut.

A lightweight, triangulated support truss structure was engineered to support the slew drive and roller screw, and to tie the Y-axis drive to the bridge structure. The main component of this structure is the roller screw support beam, which acts as the backbone for the roller screw and slew drive. The free end of the screw is radially supported by an SKF 3309 double row angular contact bearing housed within a custom pillow block. The pillow block is secured to a THK model SHS30LC linear bearing which translates along a $4.3 \mathrm{~m}$ long bearing guide rail bolted to the roller screw support beam.

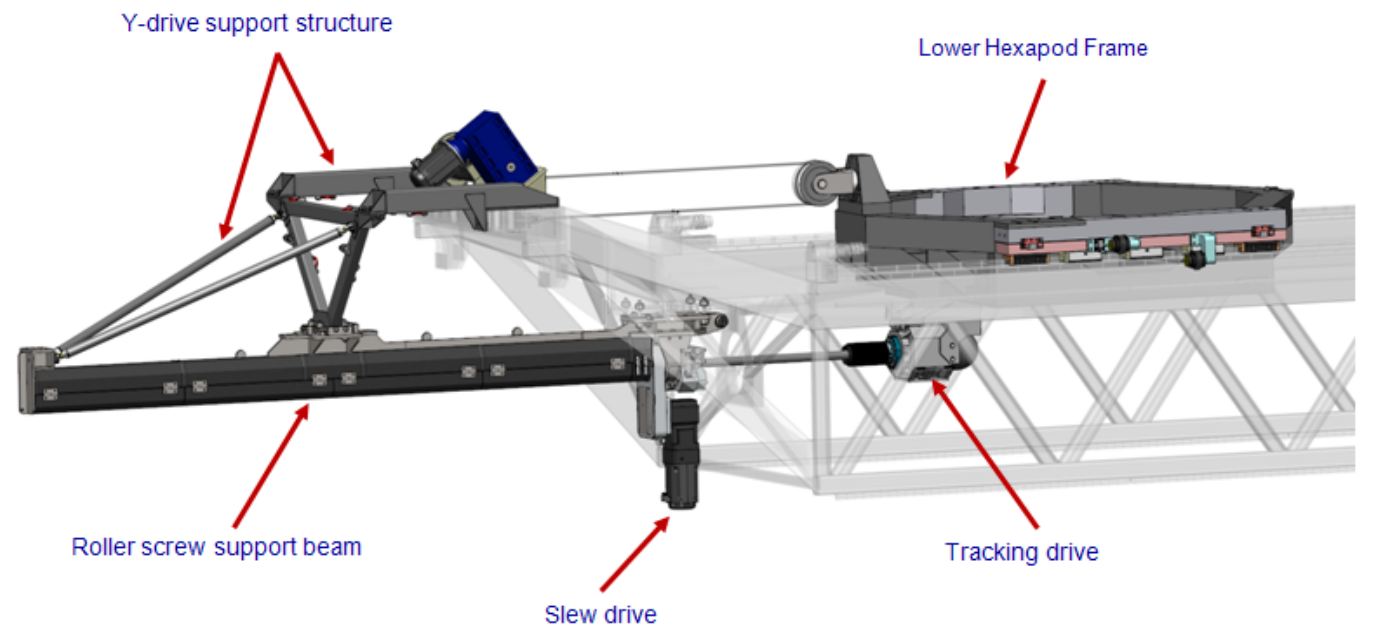

Figure 4. Y-axis drive system layout and major components. The full payload (hexapod, PFIP, WFC etc.) and telescope upper hexagon structure are hidden from view to make interfaces within the tracker bridge and LHF more apparent.

Special provisions are required to align the tracking drive (mounted to the LHF) and the slew drive and roller screw support beam (mounted to the bridge) such that all components connected by the roller screw share precise axial alignment. The difficult challenge of incorporating adjustment capability without sacrificing mounting stiffness is accomplished by dividing the 5 axes of alignment ( 5 due to the unconstrained degree of freedom in $Y$-axis) between the two major subassemblies. The primary attachment of the roller screw support beam to the bridge is via a large spherical plain bearing that is integrated into the bridge structure, which allows the beam to pivot at a point just above the slew 
drive. Adjustment hardware facilitates angular alignment of the beam in the theta, phi, and rho axes. Provisions for fine and course adjustment between the tracking drive housing and LHF permit a total $+/-10 \mathrm{~mm}$ of linear offset adjustment in the X-Z plane. Secondary adjustment provisions are incorporated between the roller screw support beam and truss attachments at its mid-section and tail to compensate for beam deflection. Essentially all of the adjustment hardware is designed to positively register with precision shims loaded in compression between metal against metal surfaces, and is deployed in such a way that each axis of adjustment is independent.

The spherical roller thrust bearing in the tracking drive is perhaps the most critical point of design. HET space constraints require positioning the screw-drive well below the X-Y plane defined by the LHF linear bearing supports. The result is a cantilevered load on the LHF structure. Deflection at the LHF tracking drive mount will naturally occur as the payload tensions the screw, in turn affecting the axial alignment between the two points supporting the screw. It is impractical to compensate for this deflection during the screw installation procedure, as the alignment would have to be performed under load and with the LHF in its deflected state. Additionally, the deflection state of the bridge is continually changing as the payload translates up and down the Y-axis. FEA analysis led to the conclusion that coupling the screw to the tracking drive and payload with a traditional duplex support bearing arrangement would be too intolerant of these two sources of static and dynamic alignment error, and the resulting reaction forces and moments imparted to the roller screw and nut would result in shortened component life. However, the same analysis shows that the self-aligning spherical roller thrust bearing is able to compensate within the range of changing alignment and reduces alignment error reaction forces to acceptable levels, therefore making this drive execution possible.

\section{TRACKER SAFETY SYSTEMS}

Scenarios have been identified, for both the existing tracker and the new tracker design, where single or simultaneous tracker system failures create the potential for uncontrolled descent of the payload down the Y-axis (runaway condition). Two of the most critical scenarios identified can be generalized as: 1) mechanical hardware failure causes complete separation of the payload from the screw-drive, and 2) an electrical power, communication, or software fault causes a control interlock failure that would allow an uncontrolled tracker move, either powered or unpowered e.g. slew and tracking disc brakes are released simultaneously while the drive motors are powered off, allowing the screw drive to back-drive freely under load. Such an event as described in case 2 has occurred on both the HET and SALT trackers and, if not forbidden in the new design, may have consequences ranging from minor to catastrophic depending upon the tracker system's mode and state at the moment of failure. Development of additional layers of failure protection and prevention, emanating from a detailed Failure Modes and Effects Analysis (FMEA), was a significant product of the Yaxis drive system design effort. Each measure is intended to limit peak mechanical shock accelerations within the WFC to $<0.5 \mathrm{~g}$ as an ideal goal, and to at least prevent impact force magnitudes that would result in unrecoverable damages in any scenario where the tracker payload reaches end-of-travel hard stops due to control loss. In addition to these qualitative criteria, crew safety and operational recovery time are paramount objective criteria.

The term "standby emergency system," as applied within this paper, refers to systems that remain idle during all normal modes of tracker operation, and which engage only after the tracker has entered a failure mode. A system may engage actively or passively and with or without fault detection awareness and still be categorized as a standby system. A redundant system is one that is continuously engaged for at least the full period, or a period beyond which the tracker is in any mode or state other than fully dormant and powered-off. Special case modes which require a deliberate override of one or both redundantly linked systems are considered rare exceptions and should be restricted with proper authority and procedure. An example and discussion of the merits of each type of safety system developed for the tracker upgrade follows.

\subsection{Standby emergency system 1: Hydraulic rail brake}

A stand-alone hydraulic emergency brake system capable of detecting and arresting a payload runaway has been developed for the new tracker. Model KB linear rail brakes from Zimmer GmbH are specifically designed to accompany linear profile bearing systems like the THK SHS series used throughout the tracker. The brakes are similar in form and dimension to the factory bearing blocks, and utilize hydraulic pressure to apply clamping force through a brake material to non-critical faces on the linear bearing guide rails. A total of six brake units are required to arrest the tracker payload with a resulting $0.4 \mathrm{~g}$ deceleration. Failsafe operation of the emergency brake system is achieved by keeping it independent and isolated from the tracker control loop. The basic hydraulic circuit is detailed in Figure 5. 


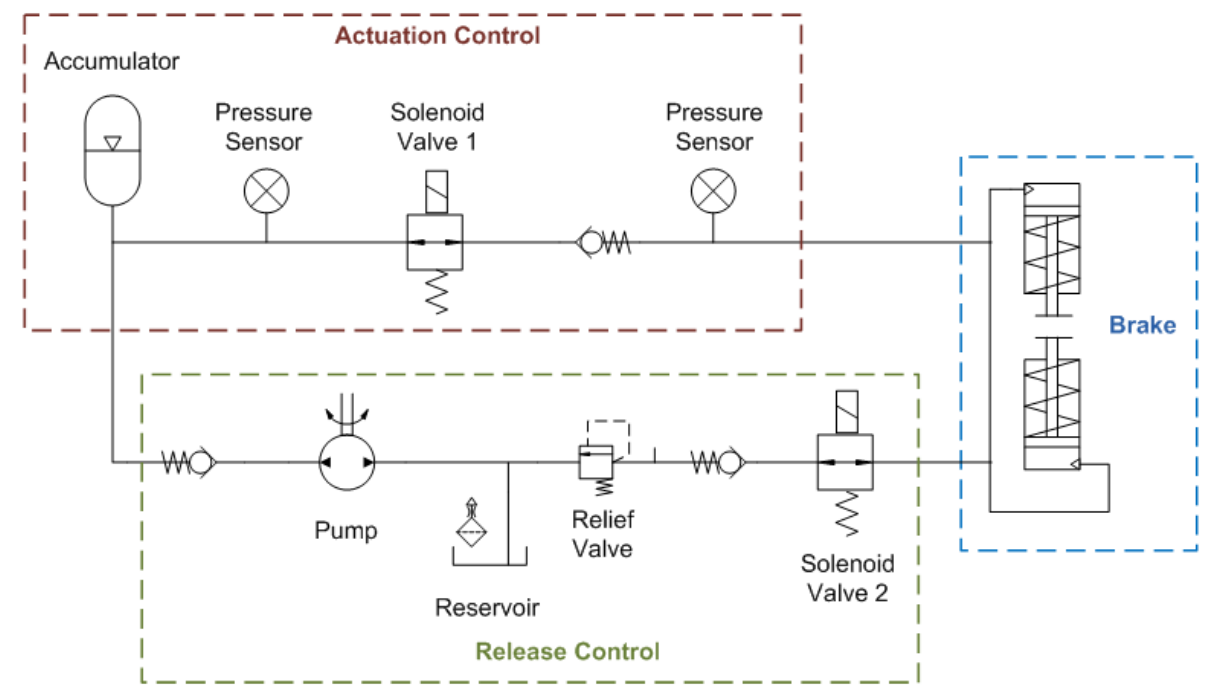

Figure 5. A pre-charged gas over oil accumulator supplies static pressure to solenoid valve 1, which requires power and signal from the brake controller to close. Overspeed detection or loss of power will open solenoid valve 1 and engage the brakes. To release the brakes, solenoid valve 1 must be energized to close and solenoid valve 2 must be energized to open (manually by a separate power circuit w/ momentary switch), which will vent brake pressure to the reservoir. An internal return spring ensures the brakes will not drag when disengaged. The brake can be cycled a limited number of times before accumulator oil must be recharged using the pump.

The hydraulic rail brake system satisfies the $0.5 \mathrm{~g}$ deceleration requirement, but raises concerns with respect to safety system health monitoring and fault detection. On a pragmatic level, such a device must be tested repeatedly and at regular intervals to assess its functional condition. On fundamental level, this is because it must change states to fulfill its intended safety purpose, and health monitoring information can only be gathered about the state which it is in currently (normal operation = "brake disengaged"). Therefore, a presumption that the brake will engage the next time it is triggered based on evidence gathered the last time it was triggered is merely statistical and not verifiable within the control system. The tracker payload will have already entered free-fall before a brake actuation failure can be detected.

\subsection{Standby emergency system 2: Energy absorbing bump stops}

Compressible aluminum honeycomb cores (Figure 6) were evaluated and tested while exploring passive safety systems to serve as backup to the emergency brake system by providing a secondary means of bringing the tracker payload to rest from free-fall without causing significant damage to the HET. Aluminum honeycomb materials are used in many applications to absorb and dissipate impact energy through uniform mechanical buckling of the cellular structure. In theory, impact with the material will generate a peak reaction force equal to that required to initiate buckling, and then the material will collapse at a lower force throughout the rest of its crush stroke. Manufacturer claims suggest that precrushing the material (pre-failing the cell walls) removes the initial force peak and that the material yields at a constant force. Several samples of different shape and size were tested in the University of Texas Center for Electromechanics (UT-CEM) laboratory to evaluate their performance. Test data shows: 1) reaction forces vary with initial impact velocity and, 2) the initial force peak is not completely mitigated by pre-crushing; therefore the dynamic crush strength of the material is not entirely independent of deformation rate (Figure 6). 

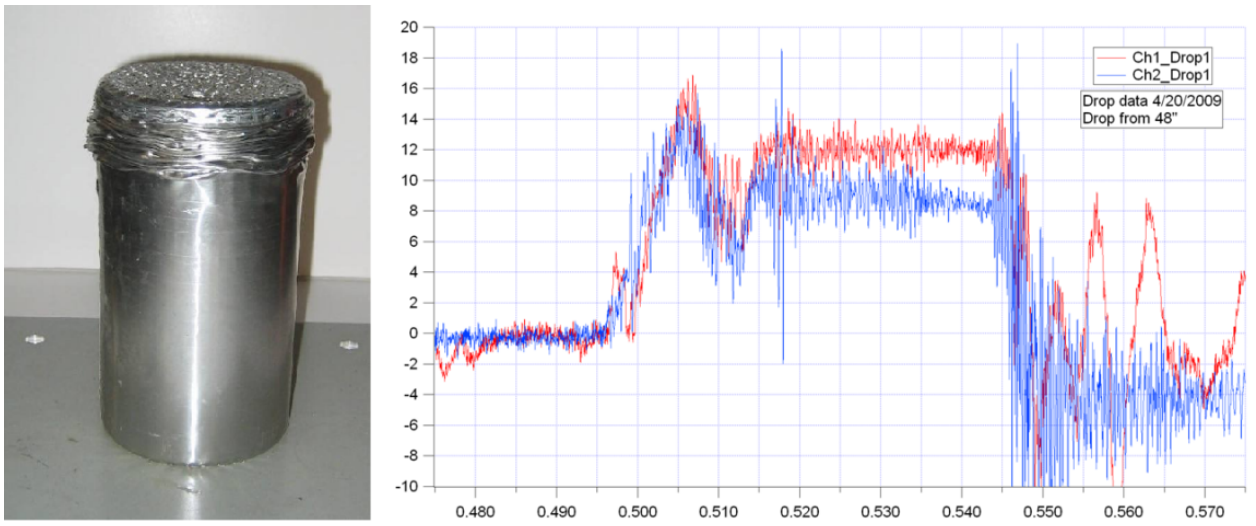

Figure 6. Left: An example of an energy absorbing, deformable bump stop. Right: A plot of acceleration (gees) versus time from tests conducted in UT-CEM's laboratory. A $450 \mathrm{~kg}$ mass guided on linear bearings was dropped onto 25,000-30,000lb rated samples from multiple elevations. The plot shows data collected from two accelerometers mounted to the test mass, and illustrates the force peak experienced upon impact.

Other attempts were made to eliminate acceleration peaks at the moment of impact such as modifying the sample geometry to taper the impact surface area, which produced some improvement. Unfortunately, the energy absorbing bump stops tested are not able to satisfy tracker performance requirements since it was determined that the overall length and stroke that would be required to reduce impact loads to acceptable levels would negatively encroach on the tracker's range of travel.

\subsection{Redundant systems}

A prime consideration for HET tracker design is how the system will transition from the normal operational mode to a failure mode. In an inherently safe design which forbids system failure due to redundancy, a system shall transition from the normal operational mode to the failure mode with essentially zero risk of serious injury to people or major damage to equipment. With either of the two standby safety systems above, a failure resulting in the runaway condition will allow the payload to be in free-fall down the slope of the tracker bridge for a fraction of time until either the emergency brake system actuates or the payload impacts the energy absorbing stops. This intermediate condition of free-fall is represented by the "Disaster Mode" in the mode diagram depicted in Figure 7. In this mode the tracker system would rely on only the emergency brake to transition the system safely to the "Failure Mode". Should the Y-brake fail to actuate in these few critical seconds it could result in the total destruction of the tracker and other parts of the telescope.

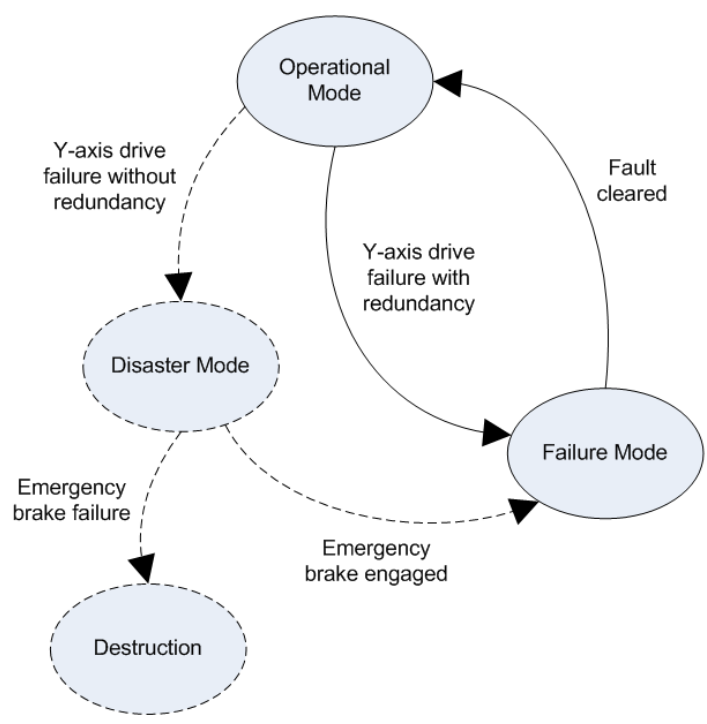

Figure 7. A standby safety system allows the tracker to enter the disaster mode before rescuing it to transition to the failure mode. A fully redundant system avoids entering the disaster mode altogether. 
Statistically, the probability of the Y-axis drive and emergency brake systems failing at the same time may be low, but the result of such a failure condition is so severe that the risk was still considered to be unacceptable. Adding yet another level of safety in case both systems fail at the same time is certainly one way to reduce risk, but the safest way to minimize the risk of disaster is to avoid entering the disaster mode in the first place. A fully capable redundant drive system, in parallel with the Y-axis drive, would provide this level of safety. The risk of entering the free fall scenario is reduced significantly. This in-effect means that the system will transition directly to the "Failure Mode" without having gone through the "Disaster Mode." The Constant Force Drive (CFD) was developed in response to these conclusions.

\section{CONSTANT FORCE DRIVE DESIGN}

\subsection{Overview of the Constant Force Drive}

The topology of the Constant Force Drive is similar to a system first deployed on SALT. A cable hoist anchored to the upper end of the bridge connects to the payload with steel wire ropes and applies constant tension in the uphill direction to offset the Y-axis vector of the payload weight (Figure 8). It is effectively an active electromechanical counterweight system working in parallel with the Y-axis screw-drive. The constant force drive is on a separate dedicated control loop which commands force in proportion to feedback from a load cell, whereas the Y-axis drive controls position based on transducer feedback as commanded by the tracker control system. A spring-damper element between the payload and load cell/wire rope provides noise isolation and smoothes response characteristics.

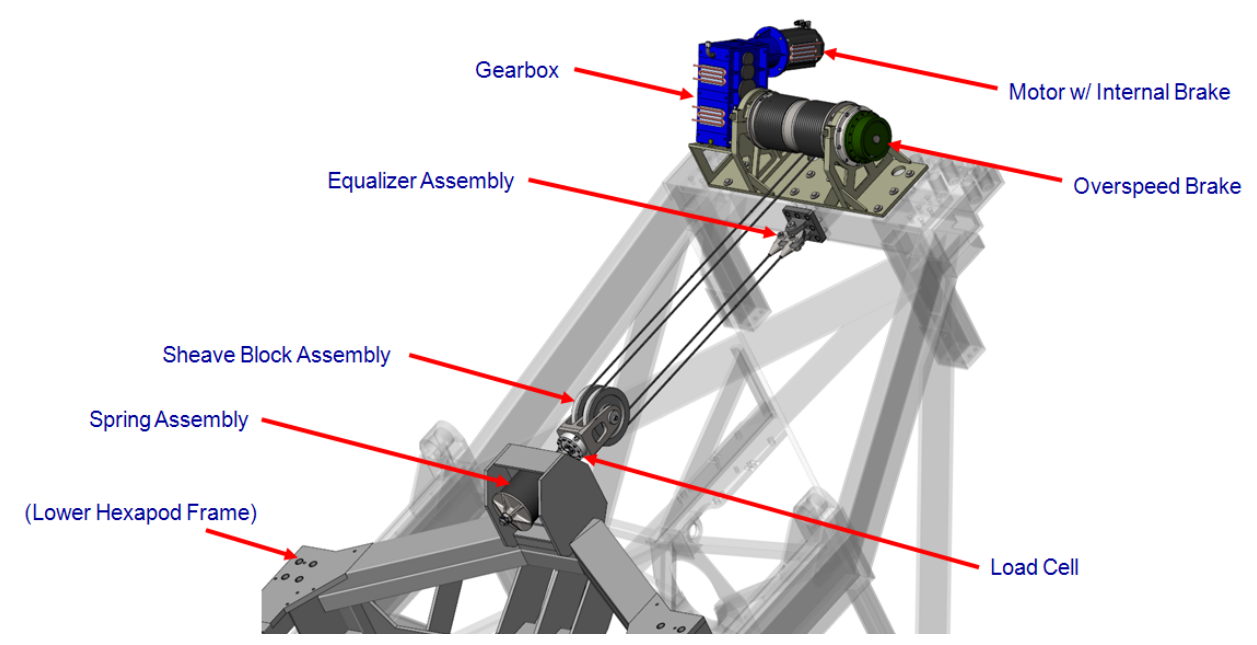

Figure 8. Constant Force Drive system layout and major components. The full payload (hexapod, PFIP, WFC etc.) and telescope upper hexagon structure are hidden from view.

\subsection{Mechanical design}

The Constant Force Drive is engineered as "fully capable" of supporting $100 \%$ of the payload weight (50 kilonewtons in the Y-axis). During normal operation, the CFD will share a predetermined component of the load, up to $90 \%$, with the $\mathrm{Y}$-axis drive. All critical components are designed with at least $5 \mathrm{x}$ factor of safety against failure. Engineering best practices for lifting hoists recommend at least 10-12x design factor for safety-critical applications, yet this would translate to impractical hardware size and weight for the HET. Additionally, this recommendation applies to a standalone system and here it is achieved within the overall tracker design factor via the pair of redundant systems.

A Danaher AKM74P motor with an integral holding bake (requires power to release) is coupled to a 140:1 reduction Renold brand HC series bevel-helical gearbox. The hoist drum has right-hand and left-hand helical grooves for single layer wire rope winding. The flanged ends of the drum, which include the output shafts, are bolted and pinned to the grooved drum core to lower replacement cost as wear from winding and unwinding the wire rope will inevitably make replacement or refurbishing necessary. The hoist drum is directly coupled to the gearbox due to space constraints, which removes a point of component failure associated with a coupling device but mandates precise alignment between the gearbox and drum shaft. The drum is supported at each end by self-aligning spherical roller bearings. The drive-side bearing is axially fixed and the non-drive side is axially floating. The non-drive side shaft of the hoist drum is coupled to a centrifugal overspeed brake from Detroit Hoist. Centrifugal brake function is fully mechanical and passive, and the 
device is friction free under normal running. When shaft rotation speed exceeds the brake's predetermined set point, the brake engages to arrest the payload within $\sim 200 \mathrm{~mm}$ stopping distance and then remains locked to hold the load.

A "two-part double" reeving configuration is used to achieve the $5 \mathrm{x}$ minimum design factor against rated break strength using half inch diameter steel wire rope. As a result, the hoist has a mechanical advantage of 2:1 and each segment of each doubled-over wire rope carries one fourth of the total tension load. Selecting this reeving arrangement and design factor has the added advantage that a single wire rope is theoretically capable of supporting the entire payload if the other wire rope fails. The wire ropes are terminated with spelter sockets (which provide a connection as strong as the wire rope itself) that attach to the equalizer assembly anchored to the bridge. As its name implies, the equalizer is a pivoting bar that compensates for differences in tension or length between the two wire ropes.

At the LHF attachment point, a sheave block and Transducer Techniques model SWP-20K fatigue-resistant load cell connect to the spring assembly pull-rod with a spherical rod-end. The pull-rod passes through a linear shaft bearing in the LHF bulkhead to compress a Firestone Industrial reinforced rubber spring.

\subsection{Key design considerations}

Optimizing the Constant Force Drive design at the system level requires careful sizing of system components. The reeving arrangement and diameter of the hoist drum determine shaft torque, rotational speed, the number turns of wire rope (and therefore drum width and rope fleet angle), and mechanical stresses in the wire rope. Several iterations were tried before reaching a balanced design with respect to system size and integration, wire rope design requirements, and torque versus speed requirements suitable for a commercially available motor and gearbox.

The choice of backdriveable versus non-backdriveable or "self-locking" gearbox was also significant. A high-ratio worm gear drive is potentially self-locking i.e. it is impossible to drive the gearbox at the input end by applying a torque, no matter how large, at the output end. A self-locking (non-backdriveable) design was preferred initially due to the inherent safety of a system that would not allow the payload to move without motive power to the gearbox. However, it was later determined that such a system would result in a gearbox efficiency of between only $50-75 \%$ and at least $15 \mathrm{~W}$ of heat dissipation at the top of the HET bridge. Furthermore, there was an unresolved risk of gearbox "stair stepping" or stickslip at very low speeds, and the HET could often require drive velocity to pass through zero in response to controller commands while following a trajectory during observation. The bevel-helical gearbox chosen is rated as $95.5 \%$ efficient. However, because it is backdriveable, speed sensing and speed limiting are required for the Constant Force Drive. A sensor was added to the drum output shaft to directly measure shaft speed and secondary speed measurement is performed indirectly through the motor. Consequently, the centrifugal overspeed brake was also added as a final failsafe to prevent payload runaway.

\subsection{Constant Force Drive and Y-axis drive control}

The Y-axis motion system on the upgraded tracker uses two drive systems operating in parallel to move the payload. These two systems have different purposes and operating methods:

- Y-axis drive - The roller screw drive system controls position

- Constant Force Drive (CFD) - The wire rope hoist system seeks to apply constant offset force

The Y-Drive employs Proportional-Integral-Derivative (PID) feedback controls to position the payload based on the feedback from an LB382 linear encoder from Heidenhain Corporation. The control algorithms also employ a feedforward control to offset static mass loads on the Y-axis drive system, but the CFD system will likely minimize the need for the feedforward terms. A secondary string potentiometer from Celesco, model PT5DC, provides lower precision absolute position feedback for system start-up control and fault monitoring.

The CFD employs an analog controller to represent PID control to the wire rope tension based on load cell output. The system also employs a spring-damper between the load cell and the payload mass carried by the CFD, necessary to ensure control stability and avoid force spikes. Using this load feedback, the CFD maintains a relatively constant force set point on the carriage at all times. Although both the Y-drive and CFD systems interact with the same load they have independent controllers. The Y-axis drive controller is integrated with the general tracker controller that is responsible for moving the other axes of the tracker, as well as all fault monitoring and Tracker Control System communication. The CFD controller is a stand-alone solid-state controller. 
Although the controllers for the Y-axis drive and the CFD are independent, they are connected via an interlock system. The purpose of this interlock is to assure that both systems are operational before $\mathrm{Y}$-axis motion may commence. CFD enabling involves two steps: 1) apply power, and 2) Ready to Operate (RTO) signal serves as the enable command. This assures that the wire ropes are tensioned as soon as the drive receives power. Both drives require the following steps to enable: 1) apply power, and 2) five parallel signals must be in the 'good' condition to send an enable (CFD RTO, Y-axis slew drive RTO, Y-axis track drive RTO, Y-axis drive controller enable, and overspeed check). The three servo-drives share a common power contactor. Therefore when power is applied, all three drives are powered in parallel.

Fault monitoring is another crucial function for the Y-drive controller. The various fault conditions monitored are:

- Following error - Excessive following error will generate a fault condition in the controller

- Overspeed - Excessive travel speed will generate a fault condition in the controller

- CFD load cell - Out of range load cell outputs will generate a fault condition in the controller

- Over-travel - Both software and limit switches continually monitor for over-travel conditions.

- Over-torque - Torque limiting couplings are used to mate the Y-drive motors to the drive screws. Proximity sensors monitor the state of these couplings. In the event of an over-torque condition a fault condition is generated.

- Over-temperature - Temperature sensors mounted on the motor housings are used to detect over-temperature conditions.

- Linear sensors - The PT5DC absolute sensors are continually compared to the LB382 sensors. A fault is generated if the indicated position from the two sensors is not the same within a certain tolerance.

A fault causes the controller to stop the motion of Y-axis drive and CFD, and engages their brakes. Even during a fault situation, the CFD will still maintain its force on the carriage.

\section{SUMMARY}

The Wide Field Upgrade for the Hobby Eberly Telescope places new demands on the telescope's structural components and drive systems. A new Y-axis positioning drive system has been developed to meet the 50kN load capacity, 4 meter travel, and $80 \mathrm{~mm} / \mathrm{second}$ speed requirements for the WFU and HETDEX. This drive system uses a translating screw supported by a lightweight truss structure, and is able to retrofit with the existing telescope structure. Telescope safety has been examined and new conclusions were reached for the HET tracker design. A viable emergency braking system was developed for the tracker, yet it revealed a fundamental issue with critical safety systems that cannot be verified as functioning at the same time the telescope is in operation. Additionally, it is vital for the safety system to transition directly to the failure mode (tracker shut-down) in the event of emergency, versus allowing the tracker to enter a potentially disastrous mode of operation before rescuing it to the failure mode. The Constant Force Drive has been developed for this purpose. This cable actuated drive functions in parallel with the Y-axis drive, and verification of its operational status is not only possible during telescope operation, it is required as a necessary condition to enter operation. 


\section{ACKNOWLEDGEMENTS}

HETDEX is led by the University of Texas at Austin McDonald Observatory and Department of Astronomy with participation from the Universitäts-Sternwarte of the Ludwig-Maximilians-Universität München, the Max-PlanckInstitut für Extraterrestriche-Physik (MPE), Astrophysikalisches Institut Potsdam (AIP), Texas A\&M University, Pennsylvania State University, and the HET consortium. In addition to Institutional support, HETDEX is funded in part by gifts from Harold C. Simmons, Robert and Annie Graham, The Cynthia and George Mitchell Foundation, Louis and Julia Beecherl, Jim and Charlotte Finley, Bill and Bettye Nowlin, Robert and Fallon Vaughn, Eric Stumberg, and many others, by AFRL under agreement number FA9451-04-2-0355, and by the Texas Norman Hackerman Advanced Research Program under grants 003658-0005-2006 and 003658-0295-2007.

\section{REFERENCES}

[1] Savage, R.D., et al., "Current status of the Hobby-Eberly Telescope wide-field upgrade", Proc. SPIE 7733-149 (2010)

[2] Hill, G. J., Gebhardt, K., Komatsu, E., Drory, N., MacQueen, P. J., Adams, J. J., Blanc, G. A., Koehler, R., Rafal, M., Roth, M. M., Kelz, A., Gronwall, C., Ciardullo, R., Schneider, D. P., "The Hobby-Eberly Telescope Dark Energy Experiment (HETDEX): Description and Early Pilot Survey Results," ASP Conf. Series, 115-118 (2008)

[3] Burge, J. H. et al, "Development of a wide-field spherical aberration corrector for the Hobby-Eberly Telescope", Proc. SPIE 7733-51 (2010)

[4] Booth, J. A., Ray, F. B., and Porter, D. S., "Development of a star tracker for the Hobby Eberly Telescope", Proc. SPIE 3351, 298-309 (1998)

[5] Worthington, M. S., Beets, T.A., Good, J. M., Mock, J. R., Murphy, B. T., South, B. J., "Design and development of a high precision high payload dual drive system", Proc. SPIE 7733-201 (2010)

[6] Ramsey, L.W., et al., "The early performance and present status of the Hobby-Eberly Telescope," Proc. SPIE 3352, 34-42 (1998)

[7] Krabbendam, V.L., Sebring, T.A., Ray, F.B. \& Fowler, J.R., "Development and performance of Hobby-Eberly Telescope 11-m segmented mirror," Proc. SPIE 3352, 436-445 (1998)

[8] Hill, G. J., MacQueen, P. J., Palunas, P., Shetrone, M. D., Booth, J. A., "Present and Future Instrumentation for the Hobby-Eberly," Proc. SPIE, 6269, 626907 (2006)

[9] Hill, G. J., Adams, J. J., Blanc, G., Booth, J. A., Chonis, T. S., Cornell, M. E., DePoy, D. L., Drory, N., Gebhardt, K., Good, J. M., Grupp, F. U., Kelz, A., Lee, H., Marshall, J. L., MacQueen, P. J., Mollison, N. T., Murphy, J. D., Rafal, M. D., "VIRUS: a massively replicated 33k fiber integral field spectrograph for the upgraded Hobby-Eberly Telescope", Proc. SPIE 7735-21 (2010)

[10] Worthington, M. S., Nichols, S. P., Good, J. M., Zierer, J. J., Mollison, N. T., Soukup, I. M., "Design and analysis of the Hobby-Eberly Telescope dark energy experiment (HETDEX) bridge", Proc. SPIE 7733-147 (2010)

[11] Zierer, J. Z., Mock, J. R., Beno, J. H., Lazzarini, P. G., Fumi, P., Anaclerio, V., Good, J. M., "The development of high-precision hexapod actuators for the Hobby-Eberly Telescope Dark Energy Experiment (HETDEX)", Proc. SPIE 7733-49 (2010)

[12] Mock, J. R., Beno, J. H., Zierer, J. J., Rafferty, T. H., Cornell, M. E., "Tracker controls development and control architecture for the Hobby-Eberly Telescope dark energy experiment", Proc. SPIE 7733-152 (2010) 